\title{
Channel Utilization and Loss Rate in a single-wavelength Fibre Delay Line (FDL) Buffer
}

\author{
B. Van Houdt*, K. Laevens ${ }^{\dagger}$, J. Lambert*, C. Blondia* and H. Bruneel ${ }^{\dagger}$ \\ *PATS Research Group, University of Antwerp, Middelheimlaan 1, B-2020 Antwerpen, Belgium \\ Email: \{benny.vanhoudt,joke.lambert,chris.blondia\}@ua.ac.be \\ ${ }^{\dagger}$ SMACS Research Group, University of Gent, St-Pietersnieuwsstraat 41, B-9000 Gent, Belgium \\ Email: \{koenraad.laevens,herwig.bruneel\}@ugent.be
}

\begin{abstract}
We present a detailed analysis of the maximum channel utilization and loss performance in an optical buffer having access to a single outgoing channel. As opposed to a conventional electronic buffer, such a system - consisting of a number of fiber delay lines - can only realize a discrete set of delays to resolve output port contention. This leads to an underutilization of the channel capacity, which reduces overall performance.

The framework considered in this paper greatly simplifies the assumptions made in previous work, which allows us to study the impact of a variety of new parameters on the performance, e.g., the burstiness of the arrival process and the correlation of consecutive burst lengths. Moreover, we present exact results for both the channel utilization and loss rate in such a system, this in contrast with all prior work which provided accurate approximations for the loss rate.

Matrix analytic methods are used to perform this analysis. To the best of our knowledge, this is the first time that such an approach is used to analyze an optical buffer. We show, amongst others, that carefully choosing the granularity parameter can, in some cases, make a substantial difference when trying to realize lower buffer losses or a high channel utilization. Optimal values of the granularity parameter are shown to be closely related to the optical burst length distribution.
\end{abstract}

\section{INTRODUCTION}

As advances in Dense Wavelength Division Multiplexing (DWDM) push fiber transmission capacities well beyond the Tbit/s, electronic switches and routers are becoming the bottlenecks of the backbone network. All-optical packet switching (OPS) could alleviate the problem. However, OPS requires practical and cost-effective implementations of optical header processing, which is still some years away. As an intermediate solution, optical burst switching (OBS) has been proposed [1], [2], [3], which avoids the need to process headers in the optical domain. Although wavelength conversion greatly reduces the need for OBS network buffering [4], contention can still arise. Resolving this contention is not straightforward due to the lack of optical RAM. The use of Fiber Delay Lines (FDLs) has been introduced to partially resolve this problem, and several architectures that make use of an FDL buffer have already been studied [5], [6], [7].

A limited number of analytic results concerning the stability and loss of such FDL based buffer systems has been published so far [8], [9], [10]. Our work consists in generalizing the results presented in [9] in a number of ways. First, we assume that new incoming optical bursts (OBs) follow a general
Markovian arrival process instead of a simple Bernoulli arrival process. Second, we allow correlation between the length of consecutive OBs, as well as correlation between the inter arrival times (IATs) and OB lengths. Finally, when studying the loss rates in a finite FDL buffer, our method allows us to produce exact analytical results, whereas all prior work relied on accurate approximations. To obtain these analytical results we make use of the matrix analytic method (MAM) which, to our best knowledge, is the first time that this method is used to analyze the performance of FDL buffers.

We focus on a single WDM channel and assume contention for it is resolved by means of an FDL buffer, which can delay, if necessary, OBs until the channel becomes available again. Unlike conventional buffers, however, it can not delay bursts for an arbitrary period of time, but only for multiples of a basic unit $D$, called the granularity of the FDLs [8], [9]. Each OB is thus either delayed $n D$ time units, for some $n=0,1, \ldots, N$, or is dropped. Hereby, $N$ is the size of the FDL buffer, the maximum achievable delay being $N D$. We assume time is slotted and we will use the duration of a single slot as the unit of time. A natural choice for the slot length would be the clock cycle in a synchronous system. Thus, for instance, the granularity $D$ would be expressed as an integer number of slots. Note that when $D$ equals one (slot), the optical buffer functions as a conventional time-slotted one, i.e., it is no longer degenerate, since then the whole range of delays (up to N) can be realized.

We will show that carefully choosing the granularity parameter $D$ can, in some cases, make a substantial difference when trying to realize low buffer losses or a high channel utilization. In general, the less uniform the burst length distribution, the more profound the impact of the granularity parameter $D$ becomes on the OB loss rate (as well as on the achievable channel utilization). Optimal values of $D$ are, in such cases, closely related to the most common OB lengths.

The paper is structured as follows. In Section II we start by presenting a stability condition for an infinite FDL buffer fed by a general Markovian arrival process. In order to improve our understanding of this stability condition, a number of special cases are considered next, e.g., Bernoulli arrivals and uncorrelated burst lengths. Within Section II we also present a number of analytical and numerical results that demonstrate which parameters influence the maximum channel utilization 


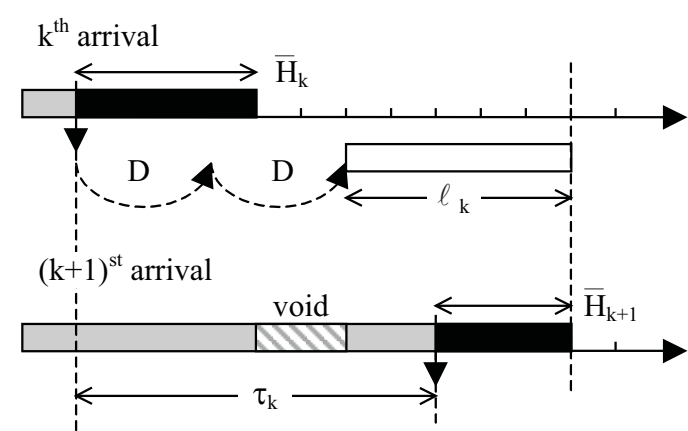

Fig. 1. Evolution of the scheduling horizon $H$ from one arrival to the next. $l_{k}$ is the length of the $k$-th OB and $\tau_{k}$ the burst IAT.

and how. Section III presents an exact method to compute the loss rate in a finite FDL buffer. A variety of numerical results, both for Bernoulli and Markovian arrivals, show that finding the optimal granularity is a delicate problem. Finally, conclusions are drawn in Section IV.

\section{STABILITY OF INFINITE FDL BUFFERS}

\section{A. General Case}

In this section we assume that the buffer is of infinite size $(N=\infty)$, so that no loss occurs. Denote by $\bar{H}_{k}$ the scheduling horizon as seen by the $k$-th arrival. It is, by definition, the earliest time (measured in slots) by which all previously arrived OBs will have left the system, and is thus the equivalent of the virtual waiting time in conventional queues. In Figure 1, the relation between $\bar{H}_{k}$ and $\bar{H}_{k+1}$ is illustrated. When the $k$-th burst sees a scheduling horizon $\bar{H}_{k}$ upon arrival, it will have to be delayed by at least that amount to avoid contention. Since the buffer is degenerate, however, this delay can not be realized exactly, the closest match being given by $D\left\lceil\bar{H}_{k} / D\right\rceil$. (Note that $\left\lceil\bar{H}_{k} / D\right\rceil$ represents the index of the FDL the $k$-th OB must traverse.) Thus, the finite granularity $D$ of the FDLs leads to voids, as illustrated in Figure 1, reducing channel utilization and lowering the system's overall capacity. The aim of this section is to obtain a simple condition to determine the maximum channel utilization $\rho_{\max }$ of a channel preceded by an infinite FDL buffer, and to determine which parameters influence $\rho_{\max }$ and how. Notice, in case of an electrical buffer $\rho_{\max }$ always equals 1 as the use of an infinite RAM buffer does not create voids.

New incoming OBs arrive according to a general Markovian arrival process [11]. Such a process is characterized by a set of $b \times b$ matrices $\left(B_{s}\right)_{s \geq 0}$, where the $(i, j)$-th element of $B_{s}$ represents the probability that the background Markov chain makes a transition from state $i$ to $j$, while, for $s>0$, a new OB with a length of $s$ time units arrives and, for $s=0$, there is no new burst arrival. Such an arrival process generally has correlated inter arrival times, as well as a correlation structure on the length of consecutive OBs, meaning that the length of an $\mathrm{OB}$ can be influenced by the size of (all) prior OBs. Moreover, the inter arrival times may also influence the length of an $\mathrm{OB}$ and vice versa.
Let $H_{n}$ be the value of the scheduling horizon at time slot $n$ and $J_{n}$ the state of the arrival process at time $n$ (for all $n \geq 0$ ). Then, $\left(H_{n}, J_{n}\right)_{n \geq 0}$ forms a discrete-time Markov chain ${ }^{1}$ (MC) with transition matrix $P$ as shown in Eq. (1). By reblocking $P$ into blocks of dimension $D b$ one finds that the $\mathrm{MC}\left(H_{n}, J_{n}\right)$ is an M/G/1 type MC with blocks of dimension $D b$ [11]. We can study the stability of the FDL buffer by investigating the ergodicity of the MC characterized by $P$. An M/G/1 type MC is known to be ergodic if and only if

$$
\pi \beta<1
$$

where $\pi$ is a left stochastic invariant vector of $A=\sum_{i \geq 0} A_{i}$ and $\beta=\sum_{i \geq 1} i A_{i} \mathbf{1}_{D b}$ (where $\mathbf{1}_{j}$ is a $j \times 1$ vector with all its entries equal to one). We can determine $\pi=\left[\pi_{1}, \pi_{2}, \ldots, \pi_{D}\right]$, where $\pi_{i}$ is a $1 \times b$ vector, explicitly as follows. Define $\bar{B}_{s}$, for $s \in\{1, \ldots, D\}$, as $\sum_{j \geq 0} B_{s+j D}$, then $A$ is found as

$A=\mathbf{1}_{D} \otimes\left[\begin{array}{llll}\bar{B}_{1} & \bar{B}_{2} & \ldots & \bar{B}_{D}\end{array}\right]+\left[\begin{array}{cccc}0 & \ldots & 0 & 1 \\ 1 & \ldots & 0 & 0 \\ \vdots & \ddots & \vdots & \vdots \\ 0 & \ldots & 1 & 0\end{array}\right] \otimes B_{0}$.

Some basic algebraic manipulations and the fact that $\theta=$ $\sum_{i=1}^{D} \pi_{i}$ show

$$
\pi_{1}=\theta \sum_{s=1}^{D} \bar{B}_{s}\left(B_{0}\right)^{s-1}\left[I-\left(B_{0}\right)^{D}\right]^{-1},
$$

where $\theta$ is the $1 \times b$ left stochastic invariant vector of $B=$ $\sum_{s \geq 0} B_{s}$. Notice, $B$ is the transition matrix of the underlying MC of the arrival process, thus, $\theta_{i}$ is the probability that the arrival process is in state $i$ at an arbitrary time instant $n$. A similar expression can be found for $\pi_{2}, \ldots, \pi_{D}$, though we do not need these to determine an expression for $\pi \beta$.

Looking at the matrices $A_{i}$ it should be clear that $\beta$ can be written as $\left[\beta_{1}^{T} \beta_{2}^{T} \ldots \beta_{2}^{T}\right]^{T}$, where $\beta_{1}$ and $\beta_{2}$ are $b \times 1$ vectors and $v^{T}$ represents the transposed of $v$. Let $\hat{B}_{s}=\sum_{j=1}^{D} B_{j+s D}$, for $s \geq 0$, then

$$
\beta_{1}=\sum_{s \geq 1} s \hat{B}_{s-1} \mathbf{1}_{b}
$$

and $\beta_{2}=B_{0} \mathbf{1}_{b}+\sum_{s>2} s \hat{B}_{s-2} \mathbf{1}_{b}$. This implies $\beta_{2}=\beta_{1}+\mathbf{1}_{b}$ as $\sum_{s \geq 0} B_{s} \mathbf{1}_{b}=\mathbf{1}_{b}$. Therefore, $\pi \beta=\pi_{1} \beta_{1}+\sum_{j=2}^{D} \pi_{j} \beta_{2}=$ $\pi_{1} \beta_{1}+\left(\theta-\pi_{1}\right)\left(\beta_{1}+\mathbf{1}_{b}\right)=\theta \beta_{1}+1-\pi_{1} \mathbf{1}_{b}$. Making use of Eq. (2), this leads us to the conclusion that the FDL buffer is stable (meaning that a burst has a finite delay with probability one) if and only if

$$
\theta \beta_{1}<\pi_{1} \mathbf{1}_{b}
$$

The left hand side $\theta \beta_{1}$ actually corresponds to $\lambda E[\lceil L / D\rceil]$, where $L$ is the $\mathrm{OB}$ length distribution ${ }^{2}$ and $\lambda$ represents the arrival rate, that is, the probability that a new incoming $\mathrm{OB}$ arrives at an arbitrary time instant $\left(\lambda=\theta \sum_{s>0} B_{s} \mathbf{1}_{b}=\theta(I-\right.$ $\left.B_{0}\right) \mathbf{1}_{b}$ ). The right hand side $\pi_{1} \mathbf{1}_{b}$ of Eq. (5) indicates what

\footnotetext{
${ }^{1}$ Notice, we observe the system at every time slot and not solely at OB arrival times.

${ }^{2} P[L=k]=\theta B_{k} \mathbf{1}_{b} /\left(\theta\left(I-B_{0}\right) \mathbf{1}_{b}\right)$
} 


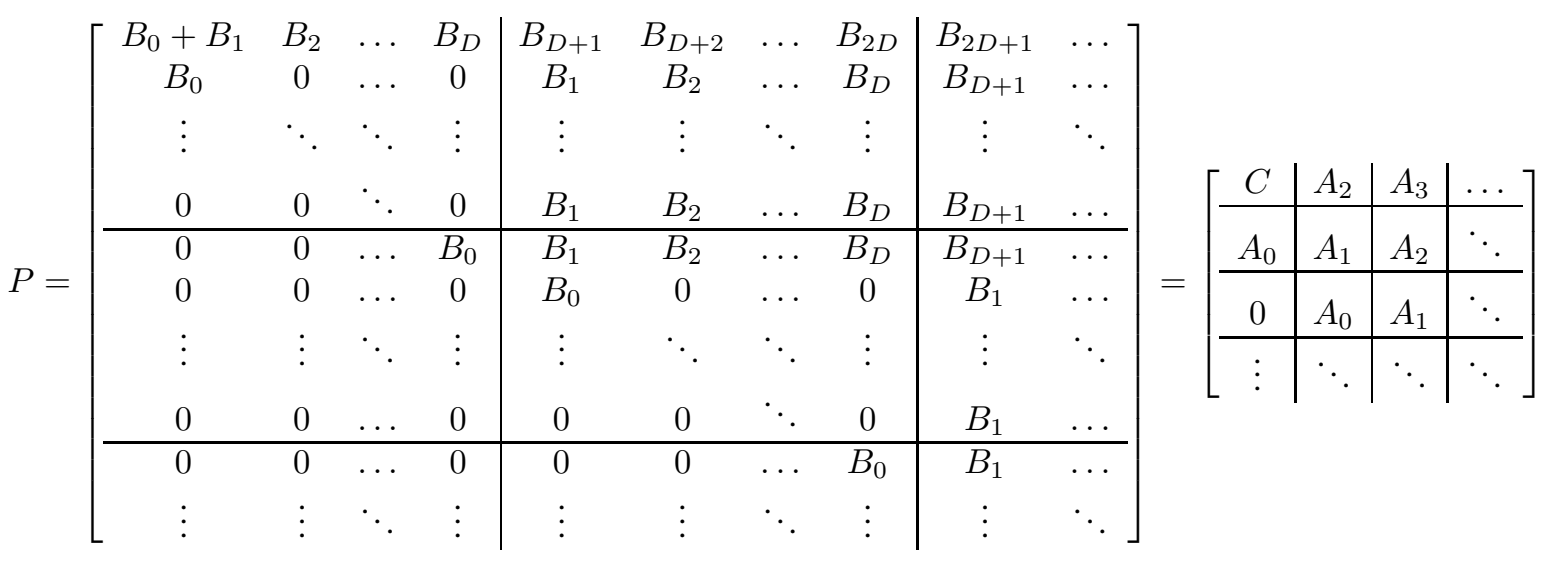

the probability would be that the scheduling horizon $H=0$ $\bmod D$ at an arbitrary time instant if $H$ had no boundary at zero.

If we approximate $\pi_{1} \mathbf{1}_{b}$ by $1 / D$ and $D E[\lceil L / D\rceil]$ by $E[L]+$ $(D-1) / 2$, we find that Eq. (5) is reduced to $\lambda(E[L]+(D-$ $1) / 2)<1$. Meaning that the maximum stable $\mathrm{OB}$ arrival rate $\lambda_{\max } \approx(E[L]+(D-1) / 2)^{-1}$, an approximation that was also proposed in [9]. Later, we demonstrate that this approximation can be very poor in certain situations. In [10] it was shown that for $D$ sufficiently small $\lambda_{\max }$ is well approximated by $(E[L]+$ $D / 2)^{-1}$ if the inter arrival times and lengths of incoming OBs are exponentially distributed. The reason for the $D / 2$ term instead of $(D-1) / 2$ is that due to the exponential IATs, the length of a void varies between 0 and $D$ as a new incoming OB need not to arrive at a slot boundary (whereas in our setup it does; hence, a void has a length of at most $D-1$ ). Next, let us try to get a better understanding of Eq. (5) by considering a few special cases.

\section{B. Bernoulli Arrivals, Uncorrelated Burst Lengths}

Assume that a new OB arrival occurs in a slot with probability $p$ independently from slot to slot, that is, the IATs are independent and geometrically distributed (with mean $1 / p$ ). Also, assume that consecutive OB lengths are uncorrelated and follow a distribution $L$. Denote $P[L \bmod D=k]$ as $l_{k}(D)$, for $k=1, \ldots, D-1$ and $P[L \bmod D=0]$ as $l_{D}(D)$. This particular scenario corresponds to the model introduced in [9].

This setup can be studied using the general model by setting $B_{s}=p P[L=s]$, for $s>0$, and $B_{0}=(1-p)$ (thus, $b=1$, $\lambda=p$ and $\theta=1$ ). As such, we can rewrite Eq. (5) as

$$
E\left[\left\lceil\frac{L}{D}\right\rceil\right]<\left(1-\bar{p}^{D}\right)^{-1} \sum_{j=1}^{D} l_{j}(D) \bar{p}^{j-1},
$$

where $\bar{p}=1-p$. Thus, the maximum input rate $p_{\max }(=$ $\left.\lambda_{\max }\right)$ for which Eq. (6) is met, is determined by $D, E[L]$ and $L \bmod D$ (because $E[\lceil L / D\rceil]$ can be written as a function of $E[L]$ and $L \bmod D$ ). This implies that many statistical properties, like for instance heavy tails, have no impact on the maximum achievable channel utilization, it is only the modulo $D$ distribution of the OB length that matters. In order

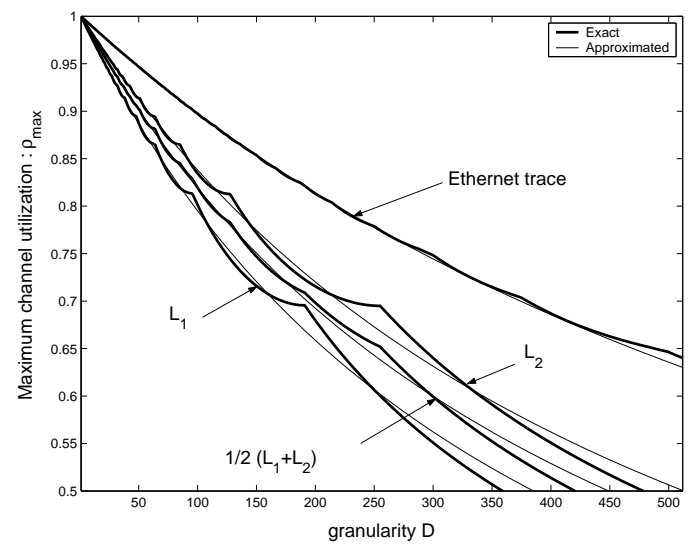

Fig. 2. Influence of the burst length distribution $L$ on $\rho_{\max }$ (Bernoulli arrivals and uncorrelated burst lengths).

to numerically solve this equation we can make use of a simple bisection algorithm: the right hand side of Eq. (6) decreases as a function of $p$ on $(0,1 / E[L]]$.

Recall, the approximation mentioned at the end of the previous subsection, reduces Eq. (6) to $p_{\max } \approx(E[L]+(D-$ $1) / 2)^{-1}$. Actually, if $L$ is distributed uniformly between 1 and $U D$, for all $U>0$, this approximation can be shown to be exact (by noticing that $l_{j}(D)=1 / D$ for all $j$ ). Figure 2 illustrates the accuracy of the approximation $p_{\max } \approx$ $(E[L]+(D-1) / 2)^{-1}$ for four different distributions: $L_{1}$ is deterministic with a mean of 192 slots, $L_{2}$ is deterministic with a mean of 256 slots, $L_{3}$ is distributed as $\left(L_{1}+L_{2}\right) / 2$ (thus, half of the bursts have a length of 192 and the other half of 256 slots) and $L_{4}$ is a burst length distribution of a typical Ethernet trace. $\rho_{\max }$ is defined as the maximum channel utilization and is calculated as $p_{\max } E[L]$. As opposed to the approximation, the exact values for $\rho_{\max }$ are not a smooth function of $D$, but contain some (minor) fluctuations. For the deterministic distributions $L_{1}$ and $L_{2}$ these occur at $(E[L]-1) / n$, for any natural number $n$. The curve for $L_{3}$ contains both the peaks of $L_{1}$ and $L_{2}$, the magnitude of these peaks has however somewhat diminished. Additional numerical experiments have shown that the peaks of a general distribution $L$ are found by 
superimposing the peaks of the deterministic distributions with means that correspond to the most frequently appearing burst lengths. Also, the more uniform the distribution $L \bmod D$, the more accurate the approximation for $p_{\max }$ seems to become, meaning that the magnitude of the peaks diminishes (which is in line with the fact that the approximation is exact if $L$ is uniformly distributed between 1 and $U D$, for some $U>0$ ). For instance, we included a typical packet length distribution obtained from an Ethernet trace ${ }^{3}$. This distribution is fairly uniform with packet lengths between 20 and 1500 bytes, but contains some very strong spikes (the most pronounced are found at 40, 576 and 1500 bytes). In Figure 2, we have set the time unit equal to $1 \mu \mathrm{sec}$ and assume that the outgoing channel has a rate of $1 \mathrm{Gbit} / \mathrm{s}$. Looking carefully at the curve for $\rho_{\max }$ we observe a peak at 300,375 and 500 , caused by the 1500 byte spike in $L$, all other peaks are too small to be visually noticed in Figure 2.

\section{Bernoulli Arrivals, Correlated Burst Lengths}

In this section, new burst arrivals still occur with a probability $p$ independently from slot to slot, the lengths of consecutive bursts, however, are no longer independent. The correlation structure on the burst lengths is characterized by a set of $l \times l$ matrices $\left(L_{k}\right)_{k \geq 1}$. If the state associated with burst $n$ is $i$, then, with probability $\left(L_{k}\right)_{i, j}$, burst $n$ has a length $k$ and the state associated with burst $n+1$ equals $j$. Thus, the probability $P[L=k]$ that an arbitrary burst has length $k$ is found as $\alpha L_{k} \mathbf{1}_{l}$, where $\alpha$ is the left stochastic invariant vector of $\sum_{k \geq 1} L_{k}$. Next, we prove that the correlation structure on the burst lengths has no influence on the stability of the system. Therefore, we can simply compute the distribution of $L$ from the matrices $L_{k}$ and apply Eq. (6) to find the maximum arrival rate $\lambda_{\max }\left(=p_{\max }\right)$ for which the FDL buffer is stable.

This particular system can be dealt with by setting $B_{s}=$ $p L_{s}$, for $s>0$, and $B_{0}=(1-p) I_{l}$, where $I_{j}$ is a unity matrix of dimension $j$ (thus, $b=l, \lambda=p$ and $\theta=\alpha$ ). Let $\bar{L}_{j}=$ $\sum_{k \geq 0} L_{j+k D}$ for $j \in\{1, \ldots, D\}$. The expression for $\pi_{1} \mathbf{1}_{l}$ can be simplified by exploiting the structure of $\left(B_{0}\right)^{j-1}=$ $(1-p)^{j-1} I_{l}$ to find

$$
\pi_{1} \mathbf{1}_{l}=p\left(1-\bar{p}^{D}\right)^{-1} \sum_{j=1}^{D}\left(\alpha \bar{L}_{j} \mathbf{1}_{l}\right) \bar{p}^{j-1} .
$$

By setting $l_{k}(D)=P[L \bmod D=k]$, for $k=1, \ldots, D-1$, and $l_{D}(D)=P[L \bmod D=0]$, one finds that the stability condition for the FDL buffer is identical to Eq. (6).

In conclusion, when new bursts arrive according to a Bernoulli process, the order in which the bursts arrive is irrelevant over any finite time scale as far as the stability of an FDL buffer is concerned. This result does not come as a surprise, because it is not difficult to show (see [10, Lemma 1]) that the length of a void between $\mathrm{OB} k$ and $k+1$ is distributed according to $\left(\tau_{k}-l_{k}\right) \bmod D$, where $\tau_{k}$ represents

\footnotetext{
${ }^{3}$ The Ethernet packet size distribution used, is based on real data traces from the Passive Network and Analysis (PNA) project conducted by the National Laboratory for Applied Network Research (NLANR).
}

the IAT between these two OBs and $l_{k}$ the length of the $k$-th OB. Thus, by putting a correlation structure on the length of consecutive OBs (which is independent from the IATs), we do not influence the distribution of the void lengths. Now, if the mean void length is unaltered, then so is $\rho_{\max }$.

\section{Markovian Arrivals, Uncorrelated Burst Lengths}

In this section we assume that $\mathrm{OB}$ lengths are uncorrelated and follow a distribution $L$. The new incoming OBs no longer form a Bernoulli process, but a general Markovian arrival process (MAP). Such a MAP can be characterized by two $d \times d$ matrices $D_{0}$ and $D_{1}$. The $(i, j)$-th entry of $D_{0}$, resp. $D_{1}$, corresponds to the probability that the underlying chain makes a transition from state $i$ to $j$, while there is no new burst arrival, resp. a new burst arrival. The arrival rate $\lambda$ equals $\gamma D_{1} \mathbf{1}_{d}$, where $\gamma=\gamma\left(D_{0}+D_{1}\right)$ and $\gamma \mathbf{1}_{d}=1$.

We can fit this model into the general framework by setting $B_{s}=D_{1} P[L=s]$, for $s>0$, and $B_{0}=D_{0}$ (thus, $b=d$ and $\theta=\gamma$ ). As such Eq. (5) can be somewhat simplified to:

$$
\lambda E\left[\left\lceil\frac{L}{D}\right]\right]<\gamma D_{1}\left(\sum_{j=1}^{D} l_{j}(D)\left(D_{0}\right)^{j-1}\right)\left(I-D_{0}^{D}\right)^{-1} \mathbf{1}_{d} .
$$

Unfortunately, there is no such thing as 'a' maximum stable arrival rate $\lambda_{\max }$ for all MAPs in general, because one can easily find two different MAPs, with rates $\lambda_{1}$ and $\lambda_{2}$, such that the FDL buffer is stable when fed by the MAP with the highest of the two rates, whereas the other MAP causes instability. This, somewhat counter intuitive result, can be explained by noticing that the void length distribution is influenced by the IAT distribution. A maximum stable input rate $\lambda_{\max }$ can be defined if we restrict ourselves to specific subclasses of the set of all MAPs, e.g., the Bernoulli arrival processes used in the previous two section, subclasses of interrupted Bernoulli processes (IBP) and many more.

In order to demonstrate the interaction between correlation and the granularity parameter $D$, we make use of the IBP process. An IBP process consists of two states: while in state 1 the IBP generates no traffic, whereas while in state 2 a new $\mathrm{OB}$ arrives with probability $p_{2}$. The sojourn time in state $i=1,2$ is geometrically distributed with a mean $s_{i}$ (hence, $\lambda=p_{2} s_{2} /\left(s_{1}+s_{2}\right)$ ). We fix $s_{1}=10 s_{2}$, meaning that an off period lasts 10 times as long as an on period (on average); hence, this traffic is very bursty. The results for less bursty setups (e.g., $s_{1}=s_{2}$ ) are similar though the impact of correlation is less pronounced. Four different values were chosen for $s_{2}$, the mean length of the on period: $1.25,28.42,807.79$ and 652530.45 slots.

Figure 3 shows the maximum channel utilization $\rho_{\max }$ (that is, $\lambda_{\max } E[L]$ ) for a deterministic distribution $L$ with a mean $E[L]$ of 256 (slots). For $s_{2}=1.25$, meaning that there is hardly any correlation, we find that $\rho_{\max }$ is close to a smoothly decreasing function ${ }^{4}$ of $D$. As in Section II-C some very mild fluctuations are observed at $(E[L]-1) / n$, with $n$ a

\footnotetext{
${ }^{4}$ Being the aforementioned approximation $1 /(1+(D-1) /(2 E[L]))$
} 


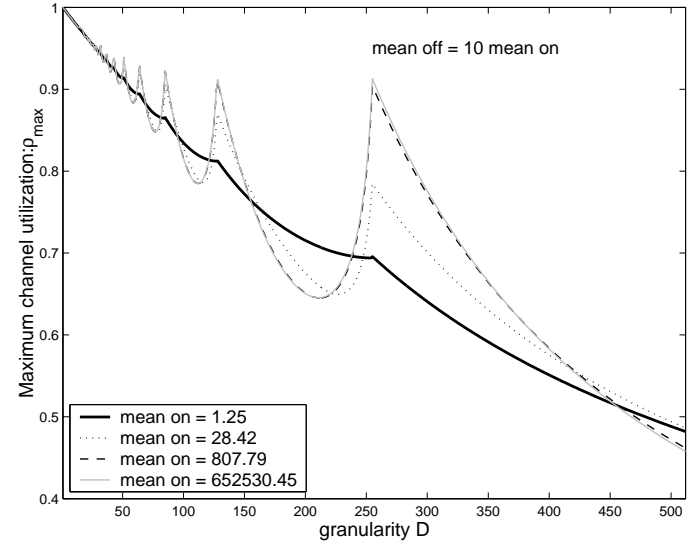

Fig. 3. Influence of correlation on $\rho_{\max }$ for deterministic distribution with mean of 256 bytes (IBP arrivals, uncorrelated burst lengths).

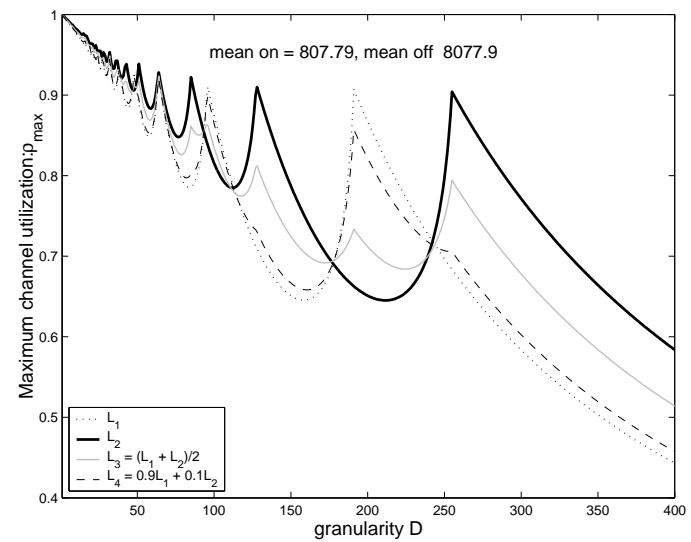

Fig. 4. Influence of the distribution $L$ on the maximum channel utilization $\rho_{\max }$ (IBP arrivals, uncorrelated burst lengths).

natural number. As the correlation increases we observe a sharp increases of $\rho_{\max }$ at the $(E[B]-1) / n$ values, whereas a decrease occurs for granularities $D$ that are located halfway between two such peaks. This can be understood by noticing that during the on period new bursts arrive fairly close to one another, thus if the OB length is a multiple of $D$ the void in between two such bursts will be limited as well. Recall, the length of the $k$-th void is distributed as $\left(\tau_{k}-l_{k}\right) \bmod D$, thus, if $l_{k}$ is close to zero modulo $D$, the size of the void will be well approximated by the IAT. On the other hand if an OB has a size of $(n+1 / 2) D$ slots for some natural number $n$, the length of the void in between bursts is often close to or above $D / 2$. Another conclusion that can be drawn from Figure 3 is that the correlation matters most over time scales that are in the order of $D$ : there is hardly any difference between the $s_{2}=807.79$ and $s_{2}=652530.45$ scenario. Thus, long term correlation has no major impact on the results. Finally, the approximation $p_{\max } \approx(E[L]+(D-1) / 2)^{-1}$ clearly becomes worse with increased correlation.

In Figure 4 we have fixed $s_{2}=807.79$ slots and considered four OB length distributions $L: L_{1}$ is deterministic with a mean of $192, L_{2}$ is deterministic with a mean of 256 (slots),
$L_{3}=\left(L_{1}+L_{1}\right) / 2$ (half of the bursts have a length of 192 , the other half a length of 256) and $L_{4}=9 L_{1} / 10+L_{2} / 10$. The location of the peaks for the $L_{3}$ and $L_{4}$ distribution are found by superimposing the location of the peaks of $L_{1}$ and $L_{2}$. Moreover, the curves for $L_{3}$ and $L_{4}$ look very much like a weighted sum of the $L_{1}$ and $L_{2}$ curves (where the weights are related to the probability that a packet has length 192 or 256 slots).

It is easy to prove that the stability condition obtained in this section is still valid even when the burst lengths are correlated, as long as there is no cross-correlation between the burst lengths and the inter-arrival times. The details are omitted for brevity.

\section{LOSS RATE IN A FINITE FDL BUFFER}

In this section we assume that the FDL buffer has a finite size $N$, thus the maximum achievable delay is $N D$ slots. OBs that see a horizon $H>N D$ when arriving, are dropped. In this section we develop an analytical method to compute the loss rate of OBs. As in Section II we assume that new OBs arrive according to a general Markov process characterized by the matrices $\left(B_{s}\right)_{s} \geq 0$.

As before, the couple $\left(H_{n}, J_{n}\right)_{n \geq 0}$ forms a discrete-time Markov chain (MC), the transition matrix of which we call $P_{N}$. Recall, $H_{n}$ is the value of the scheduling horizon at time slot $n$ and $J_{n}$ the state of the arrival process at time $n$ (for all $n \geq 0$ ). Clearly, transitions from a state of the form $(h, j)$, with $h \leq N D$, are identical to the infinite FDL system, meaning that the first $(N D+1)$ block rows of $P_{N}$ are identical to $P$ (see, Eq. (1)). If $\left(H_{n}, J_{n}\right)=\left(h, j_{1}\right)$, with $h>N D$, then $H_{n+1}=h-1$, since any new incoming OB is dropped immediately. In this case, the state of the arrival process $J_{n+1}$ equals $j_{2}$ with probability $(B)_{j_{1}, j_{2}}$.

Let $\pi^{N}=\left[\pi_{0}^{N} \pi_{1}^{N} \ldots \pi_{N D}^{N} \pi_{N D+1}^{N} \ldots\right]$ be the stochastic steady state vector of $P_{N}$, where $\pi_{i}^{N}$, for $i \geq 0$, is a $1 \times b$ vector. In order to compute $\pi_{i}^{N}$, for $i=0, \ldots, N D$, we start by censoring the MC $P_{N}$ on the set of states $\{(h, j) \mid h \leq$ $N D\}$. Given the structure of $P_{N}$ it is not hard to check that the censored chain is characterized by the transition matrix $\tilde{P}_{N}($ see $[12$, Restricted MCs]):

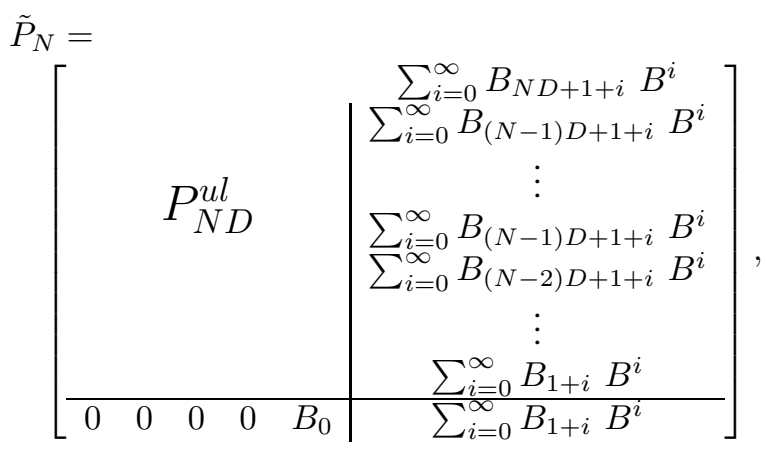

where $P_{N D}^{u l}$ represents the square matrix of dimension $N D b$ found in the upper left corner of $P$. The transition matrix $\tilde{P}_{N}$ is skip-free in one direction (since $H$ cannot decrease by more than one at a time). Therefore, we can make use of the Latouche-Jacobs-Gaver algorithm [13] to compute the 


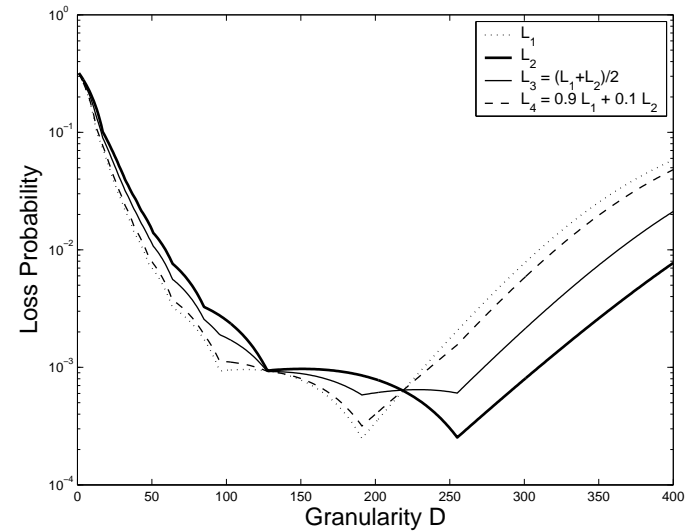

Fig. 5. Influence of the granularity $D$ on the loss probability for Bernoulli traffic with $\rho=0.5$ and $N=15$.

stochastic steady state vector $\tilde{\pi}_{N}=\left[\tilde{\pi}_{0}^{N} \ldots \tilde{\pi}_{N D}^{N}\right]$, where $\tilde{\pi}_{i}^{N}$ is a $1 \times b$ vector, in a time and memory complexity of $O\left(b^{3} N^{2} D^{2}\right)$ and $O\left(b^{2} N D\right)$, respectively. Moreover, $\tilde{\pi}_{i}^{N} c=$ $\pi_{i}^{N}$, for all $i=0, \ldots, N D$, and some constant $c$ which equals the probability that the MC characterized by $P_{N}$ has a horizon $H \leq N D$. The constant $c$ can be computed as

$$
\frac{1}{c}=1+\left[\tilde{\pi}_{0}^{N} \ldots \tilde{\pi}_{N D}^{N}\right]\left[\begin{array}{c}
r_{0}^{N} \\
\vdots \\
r_{N D}^{N}
\end{array}\right],
$$

where $r_{i}^{N}$ is a $b \times 1$ vector whose $k$-th element equals the expected number of visits that the MC characterized by $P_{N}$ makes in the set of states $\{(h, j) \mid h>N D\}$ until the first return to a state of the form $(h, j)$, with $h \leq N D$, provided that the MC started in state $(i, k)$. Looking at the structure of $P_{N}$ one finds that $r_{i}^{N}$ can be computed as

$$
r_{i}^{N}=\sum_{s>N D-\lceil i / D\rceil D+1}(s-N D+\lceil i / D\rceil D-1) B_{s} \mathbf{1}_{b} .
$$

Having found the normalization constant $c$, we can compute the loss probability from the vectors $\pi_{i}^{N}$, for $i=0, \ldots, N D$ as

$$
p_{\text {loss }}=1-\frac{\left(\sum_{i=0}^{N D} \pi_{i}^{N}\right)\left(I-B_{0}\right) \mathbf{1}_{b}}{\lambda},
$$

since the loss rate can be found as one minus the ratio between the number of new incoming OBs that are not dropped divided by the total number of new incoming OBs during an arbitrary time slot. Let us now consider the loss in an FDL buffer fed by the same arrival processes as used in previous section.

\section{A. Bernoulli Arrivals}

Assume that a new OB arrival occurs in a slot with probability $p$ independently from slot to slot, that is, the IATs are independent and geometrically distributed (with mean $1 / p$ ). Also, assume that consecutive OB lengths are uncorrelated and follow a distribution $L$. In section II-B we demonstrated that some particular choices for the granularity $D$, which depend on the OB length distribution, allow an infinite FDL buffer

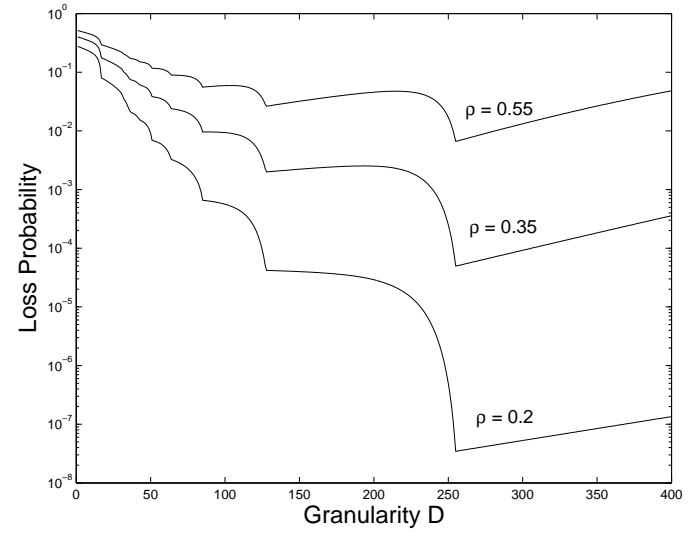

Fig. 6. Influence of the load $\rho$ on the loss probability for IBP traffic with $s_{2}=28.42, s_{1}=10 s_{2}$ and $N=15$.

to support higher traffic intensities. Figure 5 shows that these values are also good choices when considering the loss rate of a finite FDL buffer. The load $\rho=p E[B]=0.5$ for each of the curves presented in Figure 5, whereas the number of FDLs is $N=15$.

As indicated in [8], [9], there exists a trade-off between increasing the time resolution of the FDL buffer - smaller values of $D$ decrease the effect of reduced capacity due to the creation of voids - and maximum achievable delay - which, assuming $N$ is fixed, can increase only by increasing $D$. This trade-off leads to an optimal FDL granularity $D_{\text {opt }}$. For deterministic OB length distributions $L$, all our numerical experiments resulted in an optimum located at one of the values $^{5}(E[L]-1) / n$, for some integer $n$. Increasing the load $\rho$ causes $D_{\text {opt }}$ to decrease in jumps, for instance if all OBs have a length of 192 time units and $\rho=0.7$, the optimal $D$ is located at 96 (whereas for $\rho=0.5, D_{o p t}$ is found at 191). More uniform OB length distributions $L(\bmod D)$, tend to smoothen the loss probability curves and make the optimum broader (as a result, the exact value of $D_{\text {opt }}$ becomes more sensitive to and a smoother function of the offered load $\rho$ ).

\section{B. Markovian Arrivals}

In this section we assume that new incoming OBs arrive according to the IBP process defined in Section II-D. The lengths of consecutive OBs are assumed to be independent and distributed according to some distribution $L$. Figure 6 and 7 shows the influence of the offered load $\rho$ and the number of FDLs $N$ on the loss probability, respectively. In both figures the OB length distribution is chosen to be deterministic with a mean of 256 (time units). These figures illustrate that the optimal granularity $D_{\text {opt }}$ can be rather narrow when either the offered load decreases or the number of FDLs increases. For instance, for $\rho=0.2$, setting $D=225$ instead of 255 causes a difference in the loss rate of a factor 1000 . The location of this optimum seems less sensitive (though the sensitivity of the exact value of $D_{o p t}$ increases for more uniform distributions

\footnotetext{
${ }^{5}$ That is, the integer nearest to $(E[L]-1) / n$.
} 


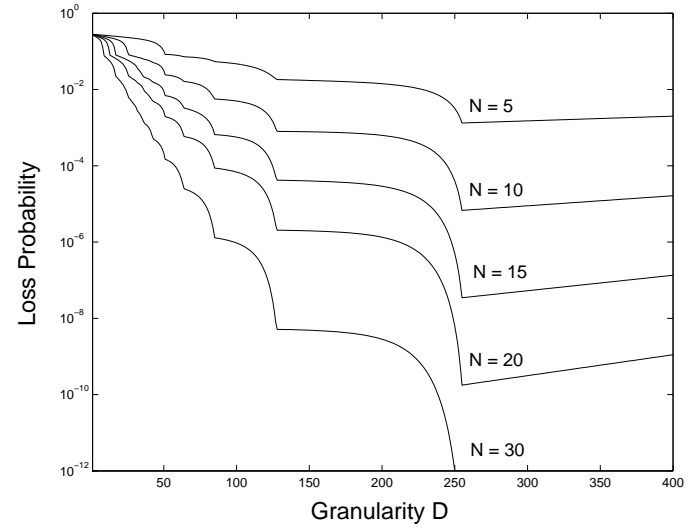

Fig. 7. Influence of the number of FDLs $N$ on the loss probability for IBP traffic with $s_{2}=28.42, s_{1}=10 s_{2}$ and $\rho=0.2$.

$L)$. Although $D_{\text {opt }}$ is always located at 255 in Figures 6 and 7, this is no longer the case if $\rho$ is further increased. One should keep in mind that the IBP process considered in Figures 6 and 7 is very bursty, because the mean length of the off period $\left(=s_{1}\right)$ is 10 times as long as the mean length of the on period $\left(=s_{2}\right)$. This implies that the offered load during the on period is 11 times as high as $\rho$. For less bursty arrival processes (e.g., $s_{1}=s_{2}$ ), we find more regular loss curves.

\section{CONCLUSIONS}

Within this paper we presented a detailed analysis of the maximum achievable channel utilization and loss rate in an optical FDL buffer. Using matrix analytic methods, we were able to study the influence of correlated inter arrival times and burst sizes, as well as cross-correlation between the two. Moreover, Eqn. (9) provided us with an exact expression for the loss probability, as opposed to earlier studies.

With respect to the maximum achievable channel utilization (MACU) the following conclusions were drawn: (i) The MACU is only affected by the burst size distribution modulo $D$, with $D$ the buffer granularity. This implies that many statistical properties, e.g., heavy tails, can be ignored. The optimal value for the granularity parameter $D$ was shown to be closely related to the modulo $D$ distribution of the burst sizes. (ii) Unless there is cross-correlation between the inter arrival times and the burst size distribution, the MACU is insensitive to possible correlation between consecutive burst sizes. (iii) Introducing correlation in the inter arrival times strongly affects the MACU and increases its sensitivity to the granularity $D$, especially when the burst size distribution is close to deterministic. However, correlation matters most over time scales that are in the order of the granularity $D$. (iv) As opposed to a classic infinite RAM buffer, increasing the burstiness of the arrival process, while keeping the mean rate fixed, can cause instability in an optical FDL buffer.

We further demonstrated that granularity values $D$ that resulted in a high MACU, are also good choices when trying to establish a low loss rate. Choosing a granularity $D$ that deviates only $10 \%$ from the optimal, can, in some extreme cases, cause the loss rate to increase by several orders of magnitude. As the burst size distribution (modulo $D$ ) becomes more uniform, the loss probability curves tend to smoothen, creating a broader optimum $D$. Increased burstiness of the arrival process has the opposite effect. Additional numerical experiments, not reported here, further showed that (i) the value of the optimal granularity $D$ is rather insensitive to the burstiness of the arrival process, as opposed to the broadness of the optimum which is strongly influenced by it. (ii) When we correlate the burst lengths with the temporary traffic intensity, the OB length distributions related to the most intense periods matter most.

In conclusion, selecting the granularity of an FDL buffer should not be taken lightly as this might severely influence the network performance.

\section{ACKNOWLEDGMENT}

The first author is a post-doctoral fellow of the FWOFlanders. This work was partly funded by the IWT-GBOU project 010058 "Optical Networking and Node Architectures".

\section{REFERENCES}

[1] J. Turner, "Terabit burst switching," Journal on High-Speed Networks, vol. 8, pp. 3-16, 1999.

[2] J. Qiao and M. Yoo, "Optical burst switching: A new paradigm for an optical Internet," Journal on High-Speed Networks, vol. 8, pp. 69-84, 1999.

[3] Y. Xiong, M. Vandenhoute, and H. Chankaya, "Control architecture in optical burst-switched WDM-networks," IEEE Journal on Selected Areas in Communications, vol. 18, pp. 1838-1851, 2000.

[4] D. Hunter and I. Andonovic, "Approaches to optical Internet packet switching," IEEE Communications Magazine, vol. 38, no. 9, pp. 116120, Sept. 2000.

[5] Z. Haas, "The staggering switch: An electronically controlled optical packet switch," Journal on Lightwave Technology, vol. 11, pp. 925-936, May/June 1993.

[6] C. et al., "CORD: Contention resolution by delay lines," IEEE Journal on Selected Areas in Communications, vol. 14, pp. 1014-1029, June 1996.

[7] D. Hunter, W. Cornwell, T. Gilfedder, A. Franzen, and I. Andonovic, "SLOB: A switch with large optical buffers for packet switching," IEEE/OSA Journal on Lightwave Technology, vol. 16, pp. 1725-1736, Oct. 1998.

[8] F. Callegati, "Optical buffers for variable length packet switching," IEEE Communications Letters, vol. 4, pp. 292-294, 2002.

[9] K. Laevens and H. Bruneel, "Analysis of a single wavelength optical buffer," in Proceedings of Infocom, San Francisco, April 2003.

[10] D. Hong, F. Poppe, J. Reynier, F. Bacelli, and G. Petit, "The impact of burstification on TCP throughput in optical burst switching networks," in Proc. of the 18th International Teletraffic Congress (ITC), Berlin (Germany), Sept. 2003.

[11] M. Neuts, Structured Stochastic Matrices of $M / G / 1$ type and their applications. New York and Basel: Marcel Dekker, Inc., 1989.

[12] G. Latouche and V. Ramaswami, Introduction to Matrix Analytic Methods and stochastic modeling. Philadelphia: SIAM, 1999.

[13] G. Latouche, P. Jacobs, and D. Gaver, "Finite markov chain models skip-free in one direction," Naval Research Logistics Quarterly, vol. 31, pp. $571-588,1984$. 